\title{
Multivariate Analysis of Matrix-Assisted Laser Desorption/Ionization Mass Spectrometric Data Related to Glycoxidation Products of Human Globins in Nephropathic Patients
}

\author{
Annunziata Lapolla, ${ }^{a}$ Eugenio Ragazzi, ${ }^{\text {b }}$ Barbara Andretta, ${ }^{\mathrm{c}}$ \\ Domenico Fedele, ${ }^{\mathrm{a}}$ Michela Tubaro, ${ }^{\mathrm{d}}$ Roberta Seraglia, ${ }^{\mathrm{d}}$ Laura Molin, ${ }^{\mathrm{d}}$ \\ and Pietro Traldi ${ }^{\mathrm{d}}$ \\ a Dipartimento di Scienze Mediche e Chirurgiche, Università di Padova, Padova, Italy \\ ${ }^{\mathrm{b}}$ Dipartimento di Farmacologia e Anestesiologia, Università di Padova, Padova, Italy \\ ${ }^{c}$ Dipartimento di Pediatria, Università di Padova, Padova, Italy \\ ${ }^{\mathrm{d}}$ CNR-ISTM, Corso Stati Uniti 4, Padova, Italy
}

To clarify the possible pathogenetic role of oxidation products originated from the glycation of proteins, human globins from nephropathic patients have been studied by matrix-assisted laser desorption/ionization mass spectrometry (MALDI), revealing not only unglycated and monoglycated globins, but also a series of different species. For the last ones, structural assignments were tentatively done on the basis of observed masses and expectations for the Maillard reaction pattern. Consequently, they must be considered only propositive, and the discussion which will follow must be considered in this view. In our opinion this approach does not seem to compromise the intended diagnostic use of the data because distinctions are valid even if the assignments are uncertain. We studied nine healthy subjects and 19 nephropathic patients and processed the data obtained from the MALDI spectra using a multivariate analysis. Our results showed that multivariate analytical techniques enable differential aspects of the profile of molecular species to be identified in the blood of end stage nephropathic patients. A correct grouping can be achieved by principal component analysis (PCA) and the results suggest that several products involved in carbonyl stress exist in nephropathic patients. These compounds may have a relevant role as specific markers of the pathological state. (J Am Soc Mass Spectrom 2007, 18, 1018-1023) ( 2007 American Society for Mass Spectrometry

$\mathrm{T}$ The role of oxidation processes occurring in vivo has been amply discussed in the literature and is considered the starting point of various complications in different diseases. In particular, it becomes relevant when it occurs on glycated proteins, leading to the production of dicarbonyl compounds. These species can react at the tissue level, leading to severe tissue modifications [1-4]. Carbonyl stress has been, and still is, the object of much experimental research devoted to the structural identification of these reactive compounds with a view to developing possible therapeutic strategies. Among the potential dicarbonyl compounds, glyoxal, methylglyoxal, and deoxyglucosone (Figure 1a) levels have been determined in many different pathological conditions and, in the case of diabetic and nephropathic patients, their levels were found to be related to the severity of the pathological condition [4-9].

Address reprint requests to Dr. Pietro Traldi, CNR-ISTM, Corso Stati Uniti 4, I35100 Padova, Italy. E-mail: pietro.traldi@adr.pd.cnr.it
It is generally believed that these products originate from glycated proteins via mechanisms generally involving the classic Maillard reaction pattern [10-13]: the hexose molecules react with the amino group that is either terminal or exposed in the protein structure, undergoing a series of dehydration and oxidation reactions that ultimately lead to the release of reactive dicarbonyls.

End-stage renal disease can be the outcome of many different kidney pathologies and is characterized by severe kidney function impairment. Some pathological events in end-stage renal disease are the consequences of an accumulation in the plasma of the so-called uremic toxins, the nature of which has yet to be fully clarified. Among these products, the activity of carbonyl compounds has recently been emphasized, and this topic has been an object of many investigations $[1,2,5,14,15]$.

Carbonyl compounds, due to their high reactivity, are believed to be responsible for various types of tissue damage related to several conditions characteristic of end-stage disease, e.g., macroangiopathy, dialysis-related 
<smiles>CC(=O)C(=O)CC(O)C(O)CC(=O)C=O</smiles>

(a)

Figure 1. (a) Molecular structures of glyoxal, methylglyoxal, and 3-deoxyglucosone and (b) structure of protein with hydroxymethyl furane moiety. amyloidosis, and myocardial damage [6, 7, 16-18]. In nonpathological states, these compounds are usually excreted by the kidney, but in the case of severe kidney disease their excretion is impaired, leading to what is called "carbonyl stress" [10, 19-23].

Previous investigations of human globins performed by MALDI mass spectrometry [24-29] were devoted mainly to ascertaining the significance of glycated hemoglobin $\left(\mathrm{HbA}_{1 \mathrm{c}}\right)$, a specific subtype of hemoglobin $\mathrm{A}$, formed by the binding of glucose to the hemoglobin molecule. Since $\mathrm{HbA}_{1 \mathrm{c}}$ degradation proceeds relatively slowly, the blood level of $\mathrm{HbA}_{1 \mathrm{c}}$ represents the average blood glucose levels of about the past four weeks. The amount of this glycated hemoglobin is used in clinics as an indicator of the average glucose blood levels, and may also suggest the amount of glycation-linked damage to tissues. MALDI mass spectrometry showed that many globin species are expressed by healthy and diabetic subjects [26-29]. Apart from the peaks related to unglycated and monoglycated $\alpha$ - and $\beta$-globins, numerous other different ionic species were identified by MALDI MS, including some glycoxidation products, reflecting the subject's oxidative stress. These studies also proved that the $\mathrm{HbA}_{1 \mathrm{c}}$ values, usually evaluated by HPLC methods or with electrospray mass spectrometric methods (LC/ESI/MS), [30] are not linearly related with the $\beta$-globin glycation level (as is generally assumed), but do correlate well with the whole pool of glycated and glycoxidated species detected by MALDI/MS [28, 29].

To clarify the question whether of oxidation products originated from the glycation of proteins survive on the protein frame or, once formed, are released from the protein, leading to the production of dicarbonyl compounds, we thought it may be of interest to evaluate the level of these substances in blood samples. Hence, our interest in the analytical data obtained by MALDI for a series of blood samples from nephropathic subjects, which we compared with the data obtained in healthy controls.

\section{Materials and Methods}

\section{Subjects}

Nineteen patients with chronic renal failure and nine normal controls were evaluated. Their clinical and metabolic characteristics are shown in Table 1. Ten of the nephropathic patients were on hemodialysis and nine were on peritoneal dialysis. All patients had been in stable clinical condition for six months before the study. Exclusion criteria were: malnutrition, smoking,

Table 1. Clinical characteristics of the patients under study (mean \pm SD)

\begin{tabular}{lccccccc}
\hline & Gender & $\begin{array}{c}\text { Age } \\
\text { (years) }\end{array}$ & $\begin{array}{c}\text { Dialysis } \\
\text { duration } \\
\text { (months) }\end{array}$ & $\begin{array}{c}\text { Fasting plasma } \\
\text { glucose }^{\mathrm{a}} \\
(\mathrm{mg} / \mathrm{dl})\end{array}$ & $\mathrm{HbA}_{1 \mathrm{c}} \mathrm{b}(\%)$ & $\begin{array}{c}\text { Blood } \\
\text { nitrogen }^{\mathrm{c}} \\
(\mathrm{mmol} / \mathrm{L})\end{array}$ & $\begin{array}{c}\text { Blood } \\
\mathrm{creatinine}^{\mathrm{d}} \\
(\mu \mathrm{mol} / \mathrm{L})\end{array}$ \\
\hline \hline $\begin{array}{c}\text { Nephropathic } \\
\text { patients }\end{array}$ & $\mathrm{F}(9) ; \mathrm{M}(10)$ & $15.6 \pm 7.8$ & $21.35 \pm 12.3$ & $76.11 \pm 20.76$ & $5.10 \pm 0.67$ & $18.58 \pm 6.56^{*}$ & $691.56 \pm 250.12^{*}$ \\
$\begin{array}{l}\text { Healthy } \\
\text { controls }\end{array}$ & $\mathrm{F}(4) ; \mathrm{M}(5)$ & $16.4 \pm 5.9$ & - & $82.96 \pm 17.73$ & $4.84 \pm 1.18$ & $5.64 \pm 3.47$ & $57.67 \pm 15.64$ \\
\hline
\end{tabular}

${ }^{*} P 0.001$, Student's t-test: calculated for nephropathic patients versus healthy controls. The difference is statistically significative only in these cases. ${ }^{a}$ Fasting plasma glucose has been obtained by a glucose-oxidase method, routinely used in clinical laboratory.

${ }^{b} \mathrm{HbA}_{1 \mathrm{c}}$ was measured by LC method, routinely used in clinical laboratory.

c,dWere evaluated by standard clinical analysis. 
diabetes mellitus, neoplasia, and chronic inflammatory diseases. All subjects gave their informed consent to the study, which was approved by the local Ethics Committee and was conducted in accordance with the Helsinki Declaration of 1975.

\section{Methods}

Fresh blood samples were diluted 1:50 in water and frozen until MALDI analysis. The samples were then diluted 1:10 with a $0.1 \%$ trifluoroacetic acid aqueous solution. The matrix solution was prepared by dissolving $10 \mathrm{mg}$ of 2,6-dihydroxyacetophenone (DHAP) (Fluka, Buchs, CH) in $1 \mathrm{~mL}$ of 1:1 (vol/vol) ethanol/ acetonitrile with the addition of $100 \mu \mathrm{L}$ of $1 \mathrm{M}$ aqueous solution of diammonium hydrogen citrate (DHAC) (Fluka). These solutions were vortexed before use. Samples were prepared by mixing equal volumes of matrix and sample solutions, applying $1 \mu \mathrm{L}$ to a stainless steel sample holder and allowing it to dry before mass spectrometric analysis.

MALDI spectra were obtained on a Voyager-DE PRO instrument (Applied Biosystems, Foster City, CA), working in positive linear mode. The target was irradiated with a $337 \mathrm{~nm}$ pulsed nitrogen laser. Ions were accelerated to $25.0 \mathrm{keV}$ kinetic energy. The working conditions for the extraction delay were: grid voltage $92 \%$; guide wire $=0.15 \%$; extraction delay time $=300$ ns. Mass spectra were obtained by averaging 50 laser shots; three separate MALDI measurements were taken for each sample to establish their reproducibility.

\section{Data Treatment and Statistical Analysis}

MALDI-MS data in the range 15 to $16.4 \mathrm{kDa}$ on the whole-blood samples of patients and healthy controls were inserted into a spreadsheet and sorted by $\mathrm{m} / \mathrm{z}$ values. The abundance of the different compounds arising from glycation and glyco-oxidation processes of $\alpha$ - and $\beta$-globins, whose identification and structures have been assigned on the basis of the Maillard reaction pattern, have been expressed as relative abundance of the corresponding globin peak, kept equal to 100. The renormalization of the spectrum so obtained has a chemical meaning; in fact $\alpha$ - and $\beta$-globins, being the two separated portion of the same precursor, must be present in molar ratio 1:1 and the difference observed in the abundance of their MALDI peaks is due to a different ionization yield of the two proteins. The data from all the subjects were converted to ASCII files by using the SpecAlign software, developed by Dr. Jason W. H. Wong, Chemistry Department, Physical and Theoretical Chemistry Laboratory, Oxford University, U.K. The software characteristics have been described elsewhere by Wong et al. [31]; for the present study, version $1.22 \mathrm{~b}$ r2 was used. Briefly, the software offers a graphical computational tool that enables simultaneous visualization and manipulation of multiple datasets; data implementation in a matrix suitable for further statistical analysis is easily possible as well as the binning of data according to a specified value; a baseline subtraction tool is also available.

The spectra were pretreated, considering only ionic species with abundance detectable over the background signal. The 15 most intense peaks across all 28 spectra (from 19 patients and nine controls) were tabulated (allowing an uncertainty of $\pm 5 \mathrm{~m} / \mathrm{z}$ in the peak positions) and their intensities collected into a $15 \times 28$ matrix. The maximum intensities within each bin were used to represent the intensity noted for that bin. Data processed in ASCII format were imported into an Excel spreadsheet and used for statistical evaluation. Further details on the spectra data handling can be found in the documentation of SpecAlign program [31].

\section{Multivariate Analysis}

Cluster analysis. Cluster analysis is a multivariate procedure of exploratory data analysis for detecting natural groupings in data and examining similarities and dissimilarities between observations. The pattern of globin glycoxidation products in patients and controls was analyzed by using a clustering procedure without any prior knowledge of grouping (unsupervised clustering) to find criteria useful in differentiating between the cases. Data were analyzed using AMADA software [32] using the K-means partition algorithm proposed by J. Hartigan [33], which splits a set of data into a selected number of groups by minimizing the sum of the squares of the distances between the data and the corresponding cluster centroid (average of the cluster members' coordinates). K-means is a clustering technique that works well if compared to hierarchical methods, and is less affected by outliers; the algorithm classifies data based on their features into $\mathrm{k}$ number of groups, $\mathrm{k}$ being a positive integer. The distances were measured with Spearman's rank correlation coefficient or Euclidean distance. In some cases, data dimensionality was reduced by correspondence analysis, a statistical ordination method, somewhat similar to principal component analysis (PCA, see below), allowing analysis of large contingency tables [34].

Principal component analysis (PCA). PCA is a mathematical procedure that reduces a large set of variables to a small set containing most of the original information. The reduced set contains what are called principal factors, which are linear combinations of the original variables. The first principal component accounts for as much of the variability in the data as possible, and each succeeding component accounts for the remaining variability. PCA can help identify new, meaningful underlying variables and can also detect clusters within multivariate data. PCA was done using the AMADA software on the matrix of $m / z$ values for all patients and controls (like cluster analysis), using a covariance matrix and standardized principal component scores. The first three components were considered for data classi- 

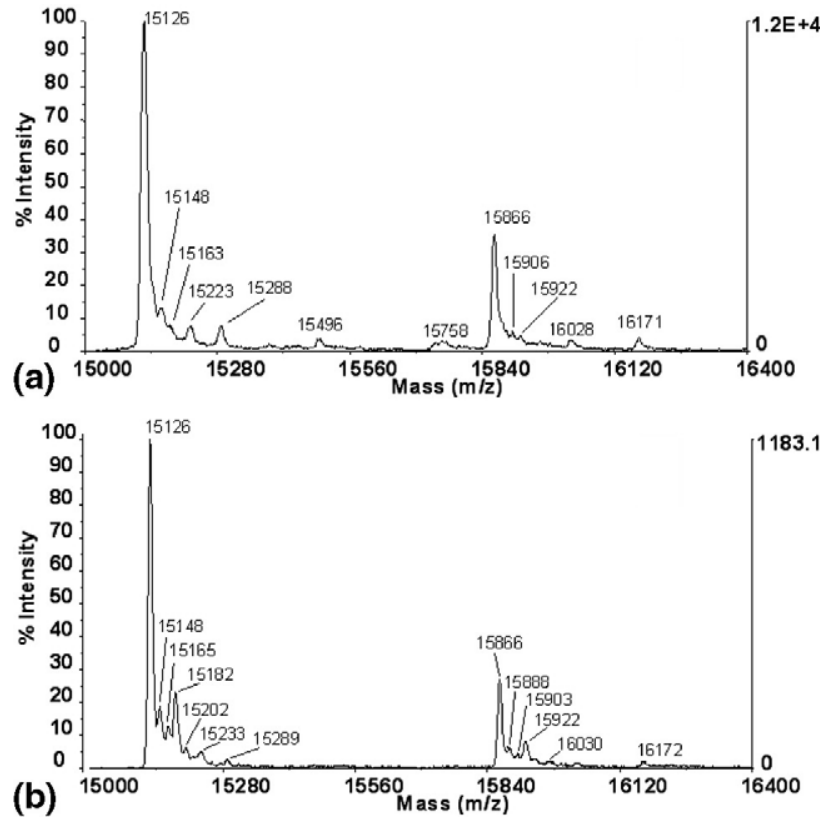

Figure 2. Typical MALDI spectra of the globin fraction from a healthy subject (a) and a nephropathic patient (b).

fication. A 3D-scatter plot was obtained with the Gnuplot 4.0 program, a freely distributed interactive data and function plotting utility for several platforms, written by Thomas Williams, Colin Kelley, et al.; http://www.gnuplot.info/index.html.

\section{Results and Discussion}

The typical MALDI spectra of the globin fraction in the range of 15 to $16.4 \mathrm{kDa}$ from a healthy subject and an end-stage renal disease patient are shown in Figure 2a and $b$, respectively. Some differences can be observed from a visual inspection of the spectra. The two most abundant peaks at $m / z 15,126$ and 15,866 are due to protonated $\alpha$ - and $\beta$-globins, respectively, but a number of other peaks are detectable. Some of them, e.g., those at $m / z \quad 15,148$ and 15,888 , do not represent different molecular species, but derive from $\mathrm{Na}^{+}$cationization of the two globins. The presence of glycated globins is clear, however, from the presence of the related peaks: the ion at $m / z 15,288$ represents the protonated glycated $\alpha$-globin, while the one at $m / z 16,028$ is due to protonated glycated $\beta$-globin. The structural assignments were tentatively done on the basis of observed masses and expectations for the Maillard reaction pattern. Consequently, they must be considered only propositive and the discussion that will follow must be considered in this view. In our opinion, this approach does not seem to compromise the intended diagnostic use of the data because distinctions are valid even if the assignments are uncertain. Further ions may derive from oxidation-dehydration processes occurring on the glycated proteins; e.g., for the ion at $\mathrm{m} / \mathrm{z} 15,223$ these processes lead to hydroxymethylfuran. In other words, these ionic species might be consistent with glycated $\alpha$-globin, which -through dehydration and oxidation taking place on the sugar moiety - might leads to hydroxymethylfurane-containing species (Figure $1 \mathrm{~b}$ ). The tall peak at $m / z 15,182$ observed in the case of a nephropathic patient (Figure 2b) might be consistent with the presence of glyoxal-containing species (Figure 1a). The analogous species is detected for $\beta$-globin at $m / z 15,922$. It is worth noting that the patients' spectra have more numerous and more abundant glycoxidated species, suggesting that more oxidation/dehydration might occur on glycated species in kidney disease.

The critical evaluation of the differences between MALDI spectra of $\alpha$ - and $\beta$-globins present in blood samples of healthy subjects and patients is a quite difficult task and consequently a multivariate analysis of the data were considered of interest.

The MALDI data of all healthy and nephropathic subjects were processed by multivariate analysis using two different approaches: one considered all species detected in the spectra, while the other excluded the data pertaining to unglycated or simply glycated $\alpha$ - and $\beta$-globins. When the $\mathrm{k}$-means clustering algorithm was applied to the spectra according to two grouping criteria, a clear differentiation between patients and controls was obtained for both including (Figure 3) and excluding (data not shown) glycated and unglycated $\alpha$ - and

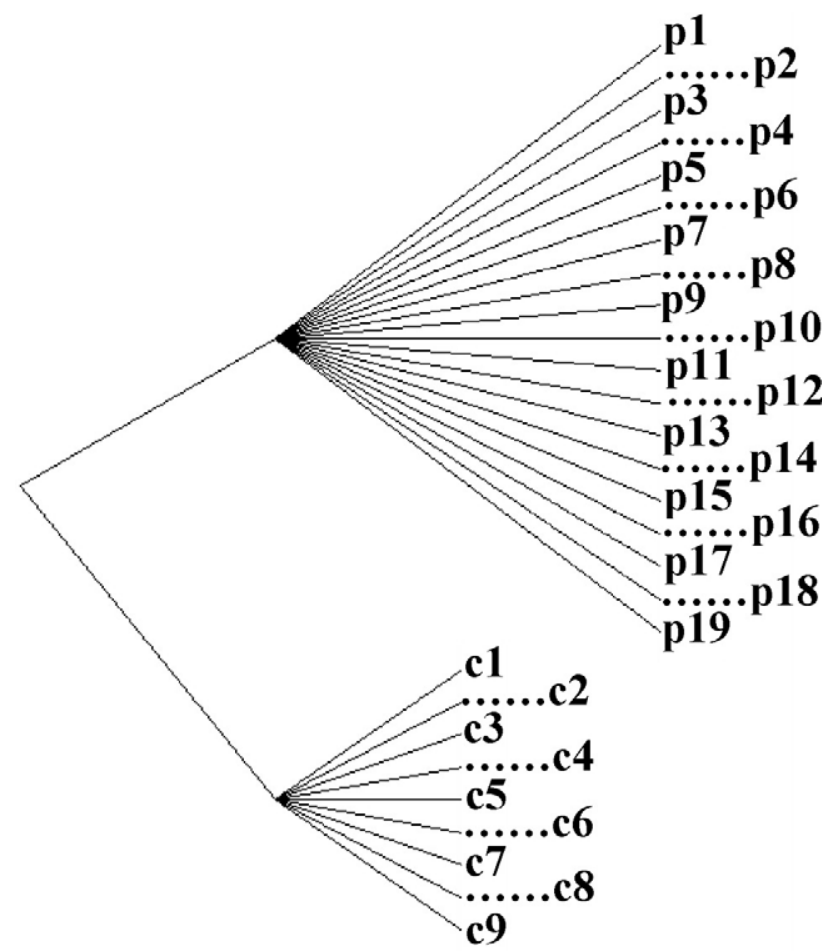

Figure 3. K-means cluster analysis of all species (after reducing data dimensionality with correspondence analysis) in the MALDI spectra of nephropathic patients (numbered as p1 to p19) and healthy controls (numbered as c1 to c9). The k number of clusters was set to 2 and Euclidean distance was used for metering. A similar grouping was also achieved with the spectra without glycated and unglycated $\alpha$ - and $\beta$-globins. 
$\beta$-globins. Using three grouping criteria on all the spectral data, the algorithm again presented one compact cluster containing all the controls, and the patients divided unevenly between the other two clusters, regardless of whether or not the data included glycated and unglycated $\alpha$ - and $\beta$-globins (data not shown). Extending the grouping criteria to four clusters applied to the whole spectra (Figure 4a) bundled the data of the controls into a single group, while the data of the patients were distributed among the other three clusters. After excluding the values for glycated and unglycated $\alpha$ - and $\beta$-globins, the clustering algorithm according to four grouping criteria led to a split in both the controls' and the patients' data (Figure $4 \mathrm{~b}$ ).

The results of the clustering procedure indicate that the MALDI pattern of the spectra can clearly differentiate patients from controls. As suggested by the cluster dendrogram obtained including peaks attributed to glycated and unglycated $\alpha$ - and $\beta$-globins (Figure 3), the contribution of these species in the spectrum appeared to have little diagnostic value in discriminating healthy subjects from nephropathic patients, whereas the pool of glycoxidated species can make this distinction.

Honing the clustering criteria, further differences relating to the glycoxidation products emerge in the patients' data that might be related to the severity of their disease and/or their reactive status. Excluding glycated and unglycated $\alpha$ - and $\beta$-globins from the cluster analysis made the patient group more homogenous, suggesting that the behavior of the globins helps to distinguish between patients, but not to separate patients from controls.

PCA confirmed the grouping of patients and controls. The 3D plots of the first three principal components are shown in Figure 5 and Figure 6. When all the species of the MALDI spectra are considered, the patients' data were quite scattered (Figure 5), but clearly distinguishable from the control group. Excluding the glycated and unglycated globins, the patients' data
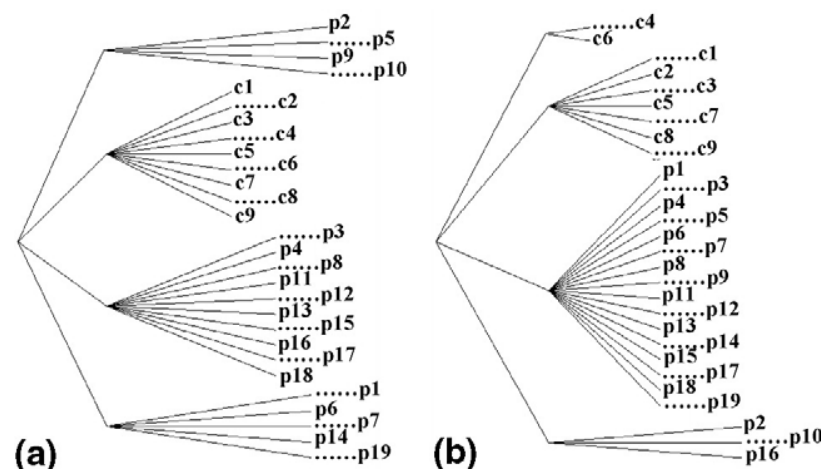

Figure 4. K-means cluster analysis of the whole species in the MALDI spectra of nephropathic patients (numbered as $\mathrm{p} 1$ to $\mathrm{p} 19$ ) and healthy controls (numbered as c1 to c9). The k number of clusters was set to 4 and Spearman's distance was used for metering. Graph (a) included and graph (b) excluded glycated and unglycated $\alpha$ - and $\beta$-globins.

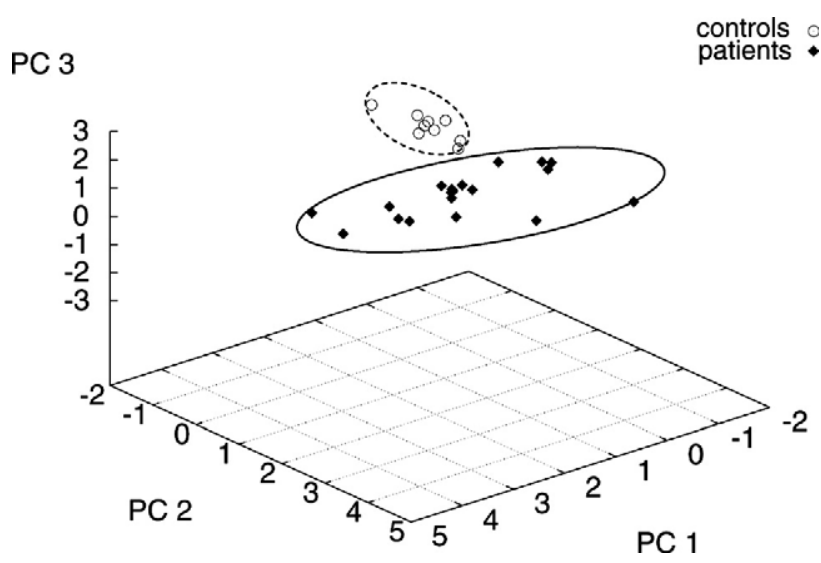

Figure 5. 3D plot of the first three components resulting from the PCA of the whole MALDI spectra of patients and controls, including glycated and unglycated $\alpha$ - and $\beta$-globins. The ovals indicate the regions of the principal component space presenting data of the two groups.

were more concentrated in a specific region of the principal component space (Figure 6). It is worth noting that the controls were well grouped in both analytical conditions, irrespective of the glycated and unglycated $\alpha$ - and $\beta$-globins. The first three components of PCA explained as much as $78 \%$ of the total variance among the data, when excluding the globin species, and $71 \%$ when including also globin species.

It is worth noting that these procedures are unsupervised, i.e., they are left free to find an aggregating criterion just on the general behavior of the native data; the results obtained, therefore, support the existence of a real capacity of the spectra to differentiate between pathological and healthy conditions.

In conclusion, the multivariate analysis used here proved capable of identifying differential aspects of the profile of ionic species from globins of end-stage nephropathic patients. The point to make is that a correct grouping can be achieved even excluding the glycated

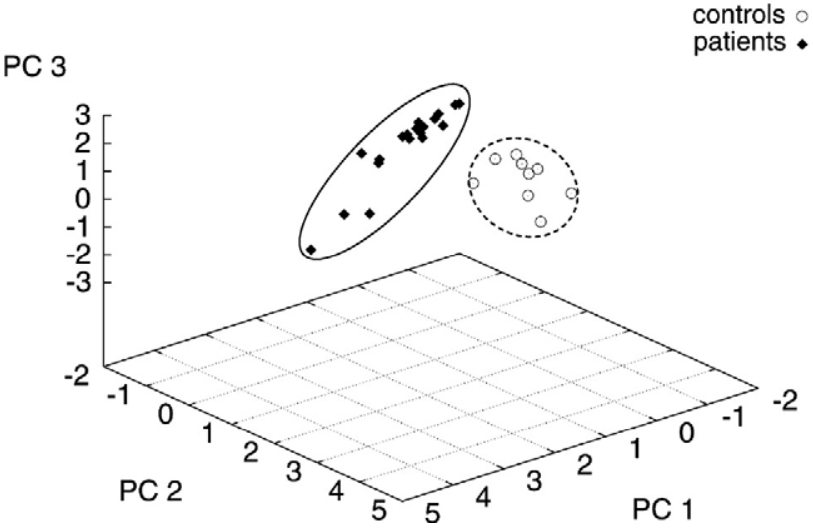

Figure 6. 3D plot of the first three components resulting from the PCA of the MALDI spectra of patients and controls, without glycated and unglycated $\alpha$ - and $\beta$-globins. The ovals indicate the regions of the principal component space presenting data of the two groups. 
and unglycated $\alpha$ - and $\beta$-globins, as confirmed by PCA as well as cluster analysis. Our data are also consistent with those of Antolini et al. [23], who suggested that PCA on oxidative stress parameters can discriminate between hemodialysis patients, transplanted patients, and controls. Our findings suggest that several products involved in the carbonyl stress are present in nephropathic patients, and that these compounds may have a relevant role as specific markers of the disease, even though they are detectable in low amounts.

\section{Acknowledgments}

The authors are grateful to Dr. Jason W. H. Wong (Chemistry Department, Physical and Theoretical Chemistry Laboratory, Oxford University, UK) for his kind help in developing the automated procedure for mass spectra data handling, as a further addition to his SpecAlign software.

\section{References}

1. Dalle-Donne, I.; Giustarini, D.; Colombo, R.; Rossi, R.; Milzani, A. Protein Carbonylation in Human Diseases. Trends Mol. Med. 2003, 9, 169-176.

2. Oberg, B. P.; McMenamin, E.; Lucas, F. L.; McMonagle, E.; Morrow, J.; Ikizler, T. A. Himmelfarb, J. Increased Prevalence of Oxidant Stress and Inflammation in Patients with Moderate to Severe Chronic Kidney Disease. Kidney Int. 2004, 65, 1009-1016.

3. Sugiyama, S.; Miyata, T.; Ueda, Y.; Tanaka, H.; Maeda, K.; Kawashima, S.; van Ypersele-de Strihou, C.; Kurokawa, K. Plasma Levels of Pentosidine in Diabetic Patients: An Advanced Glycation End Product. J. Am. Soc. Nephrol. 1998, 9, 1681-1688.

4. Miyata, T.; Saito, A.; Kurokawa, K.; van Ypersele de Strihou, C. Advanced Glycation and Lipoxidation End Products: Reactive Carbonyl Compounds-Related Uraemic Toxicity. Nephrol. Dial. Transplant. 2001, 16, 8-11.

5. Dursun, E.; Dursun, B.; Suleymanlar, G.; Ozben, T. Carbonyl Stress in Chronic Renal Failure: The Effect of Hemodialysis. Ann. Clin. Biochem. 2005, 42, 64-66

6. Bohlender, J. M.; Franke, S.; Stein, G.; Wolf, G. Advanced Glycation End Products and the Kidney. Am. J. Physiol. Renal. Physiol. 2005, 289, 645-659.

7. Kalousova, M.; Zima, T.; Tesar, V.; Dusilova-Sulkova, S.; Skrha, J. Advanced Glycoxidation End Products in Chronic Diseases-Clinical Chemistry and Genetic Background. Mutat. Res. 2005, 11, 37-46.

8. Odani, H.; Shinzato, T.; Matsumoto, Y.; Usami, J.; Maeda, K. Increase in Three $\alpha, \beta$-Dicarbonyl Compound Levels in Human Uremic Plasma: Specific in Vivo Determination of Intermediates in Advanced Maillard Reaction. Biochem. Biophys. Res. Commun. 1999, 256, 89-93.

9. Wada, T.; Miyata, T.; Kurokawa, K. Implication of Carbonyl Stress in Long-Term Uraemic Complications. Nephrol. Dial. Transplant. 1999, 14, 79-81.

10. Miyata, T.; Sugiyama, S.; Saito, A.; Kurokawa, K. Reactive Carbonyl Compounds Related Uremic Toxicity ("carbonyl stress"). Kidney Int. Suppl. 2001, 78, 25-31.

11. Sell, D. R.; Monnier, V. M. Structure Elucidation of a Senescence Cross-Link from Human Extracellular Matrix. Implication of Pentoses in the Aging Process. J. Biol. Chem. 1989, 264, 21597-21602.

12. Baynes, J. W.; Monnier, V. M., Eds; The Maillard Reaction in Aging, Diabetes and Nutrition; Alan R. Liss: New York, 1989, pp. 1-395.
13. Dyer, D. G.; Blackledge, J. A.; Thorpe, S. R.; Baynes, J. W. Formation of Pentosidine During Nonenzymatic Browning of Proteins by Glucose. Identification of Glucose and Other Carbohydrates as Possible Precursors of Pentosidine in Vivo. J. Biol. Chem. 1991, 266, 11654-11660.

14. Stopper, H.; Schupp, N.; Bahner, U.; Sebekova, K.; Klassen, A. Heidland, A. Genomic Damage in End-Stage Renal Failure: Potential Involvement of Advanced Glycation End Products and Carbonyl Stress. Semin. Nephrol. 2004, 24, 474-478.

15. Smith, A. J.; Lutgers, H. L. The Clinical Relevance of Advanced Glycation End (AGE) and Recent Developments in Pharmaceutics to Reduce AGE Accumulation. Curr. Med. Chem. 2004, 11, 2767-2784.

16. Tomizawa, H.; Yamazaki, M.; Kunika, K.; Itakura, M.; Yamashita, K. Association of Elastin Glycation and Calcium Deposit in Diabetic Rat Aorta. Diabetes Res. Clin. Pract. 1993, 19, 1-8.

17. Taniguchi, N.; Kaneto, H.; Asahi, M.; Takahashi, M.; Wenyi, C.; Higashiyama, S.; Fujii, J.; Suzuki, K.; Kayanoki, Y. Involvement of Glycation and Oxidative Stress in Diabetic Macroangiopathy. Diabetes 1996, 3 , 81-83.

18. Zoccali, C.; Mallamaci, F.; Tripepi, G. AGEs and Carbonyl Stress: Potential Pathogenetic Factors of Long-Term Uraemic Complications. Nephrol. Dial. Transplant. 2000, 15, 7-11.

19. Miyata, T.; Ueda, Y: Yamada, Y.; Izuhara, Y: Wada, T.; Jadoul, M. Saito, A.; Kurokawa, K.; van Ypersele de Strihou, C. Accumulation of Carbonyls Accelerates the Formation of Pentosidine, an Advanced Glycation End Product: Carbonyl Stress in Uremia. J. Am. Soc. Nephrol. $1998,9,2349-2356$

20. Inagi, R.; Miyata, T. Oxidative Protein Damage with Carbohydrates and Lipids in Uremia: Carbonyl stress. Blood Purif. 1999, 17, 95-98.

21. Suzuki, D.; Miyata, T.; Kurokawa, K. Carbonyl Stress. Contrib. Nephrol. 2001, 134, 36-45.

22. Vlassara, H. Advanced Glycation in Health and Disease: Role of the Modern Environment. Ann. N.Y. Acad. Sci. 2005, 1043, 452-460.

23. Antolini, F.; Valente, F.; Ricciardi, D.; Baroni, M.; Fagugli R. M. Principal Component Analysis of Some Oxidative Stress Parameters and Their Relationships in Hemodialytic and Transplanted Patients. Clin. Chim. Acta 2005, 358, 87-94.

24. Lapolla, A.; Fedele, D.; Aronica, R.; Garbeglio, M.; D'Alpaos, M. Plebani, M.; Seraglia, R.; Traldi, P. A Highly Specific Method for the Characterization of Glycation and Glycoxidation Products of Globins. Rapid Commun. Mass Spectrom. 1997, 11, 613-617.

25. Lapolla, A.; Fedele, D.; Plebani, M.; Aronica, R.; Garbeglio, M.; Seraglia, R.; D'Alpaos, M.; Traldi, P. Evaluation of Glycated Globins by MatrixAssisted Laser Desorption/Ionization Mass Spectrometry. Clin. Chem. 1999, 45, 288-290.

26. Lapolla, A.; Fedele, D.; Traldi, P. Glyco-oxidation in diabetes and related diseases. Clin. Chim. Acta 2005, 24, 236-250.

27. Lapolla, A.; Reitano, R.; Seraglia, R.; Sartore, G.; Ragazzi, E.; Traldi, P. Evaluation of Advanced Glycation end Products and Carbonyl Compounds in Patients with Different Conditions of Oxidative Stress. Mol. Nutr. Food Res. 2005, 49, 685-690.

28. Lapolla, A.; Tubaro, M.; Fedele, D.; Reitano, R.; Arico N. C.; Ragazzi, E.; Seraglia, R.; Vogliardi, S.; Traldi, P. A Matrix-Assisted Laser Desorption/Ionization Mass Spectrometry Study of the Nonenzymatic Glycation Products of Human Globins in Diabetes. Rapid Commun. Mass Spectrom. 2005, 19, 162-168.

29. Lapolla, A.; Tubaro, M.; Reitano, R.; Arico N. C.; Ragazzi, E.; Seraglia, R.; Vogliardi, S.; Traldi, P.; Fedele, D. The Complexity of Nonenzymatic Glycation Product Sets of Human Globins. Diabetologia 2005, 47, 1712 1715 .

30. Roberts, N. B.; Green, B. N.; Morris, M. Potential of Electrospray Mass Spectrometry for Quantifying Glycohemoglobin. Clin. Chem. 1997, 43, 771-778.

31. Wong, J. W. H.; Cagney, G.; Cartwright, H. M. SpecAlign-Processing and Alignment of Mass Spectra Datasets. Bioinformatics 2005, 21, 2088 2090.

32. Xia, X.; Xie, Z. AMADA: Analysis of Microarray Data. Bioinformatics 2001, 17, 569-570.

33. Hartigan, J.; Wong, M. A. K-Means Clustering Algorithm. Applied Statistics 1979, 28, 100-108.

34. Greenacre, M. J. Correspondence Analysis in Practice, 1st ed.; Academic Press: London, 1993, pp. 1-256. 